\title{
On Fujita invariants of subvarieties of a uniruled variety
}

\author{
Christopher D. Hacon and Chen Jiang
}

\begin{abstract}
We show that if $X$ is a smooth uniruled projective variety and $L$ is a big and semiample $\mathbb{Q}$-divisor on $X$, then there exists a proper closed subset $W \subset X$ such that every subvariety $Y$ with Fujita invariant $a(Y, L)$ greater than $a(X, L)$ is contained in $W$.
\end{abstract}

\section{Introduction}

If $X$ is a smooth projective variety and $L$ is a big $\mathbb{Q}$-divisor on $X$, then the Fujita invariant, or $a$-constant, is defined as follows:

$$
a(X, L)=\inf \left\{t>0 \mid K_{X}+t L \text { is big }\right\} .
$$

Note that $a(X, L) \in \mathbb{R}_{\geqslant 0}$ is well defined since $K_{X}+t L$ is big for all $t>0$ sufficiently large and that $a(X, L)>0$ if and only if $K_{X}$ is not pseudo-effective. It is easy to see that the $a$-constant is a birational invariant in the sense that if $\nu: X^{\prime} \rightarrow X$ is a birational morphism of smooth varieties and $L^{\prime}=\nu^{*} L$, then $a(X, L)=a\left(X^{\prime}, L^{\prime}\right)$ (cf. [HTT15, Proposition 2.7]). Therefore, we may also define the $a$-constant for a big $\mathbb{Q}$-Cartier $\mathbb{Q}$-divisor $L$ on an arbitrary projective variety $X$ by setting

$$
a(X, L):=a\left(X^{\prime}, L^{\prime}\right),
$$

where $\nu: X^{\prime} \rightarrow X$ is a resolution of singularities and $L^{\prime}=\nu^{*} L$. Note that if $X$ is smooth, then the $a$-constant is the usual pseudo-effective threshold; however, if $X$ is singular, these numbers may be different.

A conjecture of Batyrev and Manin relates arithmetic properties of varieties with ample anticanonical class to geometric invariants such as $a$-constants. Roughly speaking, this conjecture predicts that the asymptotic behavior of a point-counting function is controlled by two geometric invariants known as the $a$-constant and the $b$-constant. In view of this conjecture, it is expected that almost all subvarieties of a uniruled variety $X$ should have $a$-constants not greater than that of $X$. See [HTT15, LTT14] for more background on the Batyrev-Manin conjecture.

In [LTT14], $a$-constants were intensively studied by Lehmann, Tanimoto and Tschinkel, motivated by the conjecture of Batyrev and Manin. They show that if $X$ is a smooth uniruled

Received 13 July 2016, accepted in final form 3 August 2016.

2010 Mathematics Subject Classification 14C20.

Keywords: Fujita invariant, uniruled variety.

This journal is (C) Foundation Compositio Mathematica 2017. This article is distributed with Open Access under the terms of the Creative Commons Attribution Non-Commercial License, which permits non-commercial reuse, distribution, and reproduction in any medium, provided that the original work is properly cited. For commercial re-use, please contact the Foundation Compositio Mathematica.

The first author was supported by NSF research grants nos. DMS-1300750 and DMS-1265285 and by a grant from the Simons Foundation, award number 256202. The second author was supported by JSPS KAKENHI grant number JP16K17558, the Program for Leading Graduate Schools and the World Premier International Research Center Initiative (WPI), MEXT, Japan. 


\section{ON FUJITA INVARIANTS}

projective variety and $L$ is an ample $\mathbb{Q}$-divisor on $X$, then there exists a countable union of proper closed subsets $W \subset X$ such that every subvariety $Y$ satisfying $a(Y, L)>a(X, L)$ is contained in $W$ [LTT14, Theorem 1.1]. For the purpose of applications, it is expected that one may choose $W$ to be a proper closed subset of $X$. The purpose of this note is to prove that this is indeed the case.

Theorem 1.1. Let $X$ be a smooth uniruled projective variety and $L$ a big and semiample $\mathbb{Q}$ divisor on $X$. Then there exists a proper closed subset $W \subset X$ such that every subvariety $Y$ satisfying $a(Y, L)>a(X, L)$ is contained in $W$.

Note that this result is proven in [LTT14, Theorem 1.2] assuming that a weak version of the BAB conjecture (due to Borisov, Borisov and Alexeev) holds in dimension $n-1=\operatorname{dim} X-1$. We expect that Theorem 1.1 also holds if we just assume that $L$ is big and nef (rather than big and semiample).

Our idea is to replace the WBAB conjecture assumed in [LTT14, Theorem 1.2] by constructing non-klt centers (see Definition 2.5 and Proposition 2.8) and applying the finiteness of the $a$ constants (see Corollary 2.15). This is an application of a recent result of Di Cerbo [DiC17] based on a boundedness result proved by Birkar [Bir16].

\section{Preliminaries}

In this paper, we work over the field of complex numbers $\mathbb{C}$.

\subsection{Facts on $a$-constants}

In this subsection, for the convenience of the reader, we collect several facts about $a$-constants that were proven in [LTT14].

Proposition 2.1 ([LTT14, Proposition 4.1]). Let $X$ be a smooth projective variety and $L$ a big and nef $\mathbb{Q}$-divisor. Let $\mathcal{U} \rightarrow W$ be a family of subvarieties of $X$ such that $\mathcal{U} \rightarrow X$ is dominant. Then a general member $Y$ of the family $\mathcal{U}$ satisfies $a(Y, L) \leqslant a(X, L)$.

Theorem 2.2 ([LTT14, Theorem 4.2]). Let $X$ be a smooth projective variety and $L$ a big and nef $\mathbb{Q}$-divisor. Let $\pi: \mathcal{U} \rightarrow W$ be a family of subvarieties of $X$. There exists a proper closed subset $V \subset X$ such that if a member $Y$ of the family $\mathcal{U}$ satisfies $a(Y, L)>a(X, L)$, then $Y \subset V$.

Proposition 2.3 ([LTT14, Proposition 4.6]). Let $X$ be a smooth uniruled projective variety and $L$ a big and nef $\mathbb{Q}$-divisor. Then either

(i) $X$ is covered by proper subvarieties $Y$ satisfying $a(Y, L)=a(X, L)$ or

(ii) $X$ is birational to a $\mathbb{Q}$-factorial terminal Fano variety $X^{\prime}$ of Picard number 1.

Lemma 2.4 ([LTT14, Lemma 4.7]). Let $X$ be a smooth projective variety and $L$ a big and nef $\mathbb{Q}$-divisor on $X$. Fix a constant $C$. Then the subset of Chow $(X)$ parametrizing subvarieties of $X$ that are not contained in $\mathbf{B}_{+}(L)$ and are of $L$-degree at most $C$ is bounded.

\subsection{Non-klt centers}

We follow the standard notation and conventions of the minimal model program; see, for example, [Kol97]. 


\section{D. HACON AND C. JiAng}

Definition 2.5. Let $(X, \Delta)$ be a pair with $X$ a normal variety and $\Delta$ an effective $\mathbb{Q}$-divisor such that $K_{X}+\Delta$ is $\mathbb{Q}$-Cartier. We say that a subvariety $V \subset X$ is a non-klt center of $(X, \Delta)$ if it is the image of a divisor of discrepancy at most -1 . We will denote by $\operatorname{Nklt}(X, \Delta)$ the union of all non-klt centers of $(X, \Delta)$, which is a proper closed subset of $X$. A non-klt place is a valuation corresponding to a divisor of discrepancy at most -1 . A non-klt center $V$ is pure if $K_{X}+\Delta$ is $\log$ canonical at the generic point of $V$. If, moreover, there is a unique non-klt place lying over the generic point of $V$, we will say that $V$ is an exceptional non-klt center.

The following is a weak form of Kawamata's subadjunction theorem.

Theorem 2.6 (Subadjunction; see [Jia13, Proposition 5.1]). Let $V \subset X$ be a non-klt center of a pair $(X, \Delta)$ which is log canonical at a general point of $V$. Let $\nu: V^{\nu} \rightarrow V$ be the normalization. Then there is an effective $\mathbb{Q}$-divisor $\Delta_{V^{\nu}}$ on $V^{\nu}$ such that

$$
\left.\nu^{*}\left(K_{X}+\Delta\right)\right|_{V_{\nu}} \sim_{\mathbb{Q}} K_{V^{\nu}}+\Delta_{V^{\nu}}
$$

We have the following connectedness lemma of Kollár and Shokurov for the non-klt locus (cf. Shokurov [Sho93, Sho94], Kollár [Kol92, Theorem 17.4]).

Theorem 2.7 (Connectedness lemma). Let $f: X \rightarrow Z$ be a proper morphism of normal varieties with connected fibers and $D$ a $\mathbb{Q}$-divisor such that $-\left(K_{X}+D\right)$ is $\mathbb{Q}$-Cartier, $f$-nef and $f$-big. Write $D=D^{+}-D^{-}$, where $D^{+}$and $D^{-}$are effective with no common components. If $D^{-}$ is $f$-exceptional (that is, all of its components have image of codimension at least 2), then $\operatorname{Nklt}(X, D) \cap f^{-1}(z)$ is connected for any $z \in Z$.

We can use the following proposition to construct non-klt centers.

Proposition 2.8 (cf. [Lai16, Lemma 3.2]). Let $X$ be a $\mathbb{Q}$-factorial terminal Fano variety of dimension $n$. Assume $\left(-K_{X}\right)^{n}>(w n)^{n}$ for some positive rational number $w$. Then for every point $p \in X$, there is an effective $\mathbb{Q}$-divisor $\Delta_{p} \sim_{\mathbb{Q}}-\frac{1}{w} K_{X}$ such that the unique minimal non-klt center $V_{p} \subset \operatorname{Nklt}\left(X, \Delta_{p}\right)$ containing $p$ is exceptional.

Proof. Fix a point $p$. Fix a positive rational number $w^{\prime}$ such that $\left(-K_{X}\right)^{n}>\left(w^{\prime} n\right)^{n}>(w n)^{n}$. By [Kol97, Theorem 6.7.1], there is an effective $\mathbb{Q}$-divisor $\Delta_{p}^{\prime} \sim_{\mathbb{Q}}-\frac{1}{w^{\prime}} K_{X}$ such that $\left(X, \Delta_{p}^{\prime}\right)$ is not $\log$ canonical (lc) at $p$. Let $0<t \leqslant 1$ be the unique rational number such that $\left(X, t \Delta_{p}^{\prime}\right)$ is lc but not klt at $p$. By [Amb98, Proposition 3.2, Lemma 3.4], we can find an effective $\mathbb{Q}$-divisor $M_{p} \sim_{\mathbb{Q}}-\frac{1}{w^{\prime}} K_{X}$ and some rational number $a>0$ such that for any rational number $0<\epsilon \ll 1$, the pair $\left(X,(1-\epsilon) t \Delta_{p}^{\prime}+\epsilon a M_{p}\right)$ has a unique minimal non-klt center $V_{p}$ passing through $p$ which is exceptional. Note that

$$
(1-\epsilon) t \Delta_{p}^{\prime}+\epsilon a M_{p} \sim_{\mathbb{Q}}-\frac{(1-\epsilon) t+\epsilon a}{w^{\prime}} K_{X}
$$

and $((1-\epsilon) t+\epsilon a) / w^{\prime}<1 / w$ for $0<\epsilon \ll 1$. Since $-K_{X}$ is ample, by adding a $\mathbb{Q}$-divisor $\mathbb{Q}$-linearly equivalent to a multiple of $-K_{X}$ to $\Delta_{p}^{\prime}$, we conclude that there exists an effective $\mathbb{Q}$-divisor $\Delta_{p}$ such that $\Delta_{p} \sim_{\mathbb{Q}}-\frac{1}{w} K_{X}$ and $\left(X, \Delta_{p}\right)$ has a unique minimal non-klt center $V_{p}$ passing through $p$ which is exceptional.

Lemma 2.9. We keep the notation of Proposition 2.8. If $w>2$, then $\operatorname{dim} V_{p}>0$ for a general point $p$.

Proof. Assume to the contrary that there exist $p_{1} \in X$ such that $V_{p_{1}}=\left\{p_{1}\right\}$ and $p_{2} \in$ $X \backslash \operatorname{Supp}\left(\Delta_{p_{1}}\right)$ such that $V_{p_{2}}=\left\{p_{2}\right\}$. Then $p_{1}$ and $p_{2}$ are contained in $\operatorname{Nklt}\left(X, \Delta_{p_{1}}+\Delta_{p_{2}}\right)$ and $p_{2}$ 


\section{ON FUJITA INVARIANTS}

is isolated by construction. On the other hand,

$$
-\left(K_{X}+\Delta_{p_{1}}+\Delta_{p_{2}}\right) \sim_{\mathbb{Q}}\left(1-\frac{2}{w}\right)\left(-K_{X}\right)
$$

is ample. By the connectedness lemma, $\operatorname{Nklt}\left(X, \Delta_{p_{1}}+\Delta_{p_{2}}\right)$ is connected, which gives a contradiction.

\subsection{Finiteness of $a$-constants}

We recall the main result of [DiC17] in this subsection.

Definition 2.10. Let $X$ be a normal projective variety and $H$ a big $\mathbb{Q}$-divisor. We define the pseudo-effective threshold to be

$$
\tau(X, H):=\inf \left\{t \geqslant 0 \mid K_{X}+t H \text { is big }\right\} .
$$

Note that if $X$ is smooth, the $a$-constant and pseudo-effective thresholds coincide.

Definition 2.11 (cf. [DiC17, Definition 3.1]). Fix a positive integer $n$ and two positive real numbers $\epsilon$ and $\delta$. We define $\mathcal{D}_{n}(\epsilon, \delta)$ to be the set of lc pairs $(X, \Delta)$ such that

(i) $X$ is a normal projective variety of dimension $n$,

(ii) $\Delta$ is a big $\mathbb{Q}$-divisor with coefficients $\geqslant \delta$, and

(iii) $(X, t \Delta)$ is $\epsilon$-lc and $K_{X}+t \Delta$ is pseudo-effective for some $0 \leqslant t \leqslant 1$.

Definition 2.12 (cf. [DiC17, Definition 3.2]). Fix a positive integer $n$ and two positive real numbers $\epsilon$ and $\delta$. We define the set

$$
\mathcal{T}_{n}(\epsilon, \delta):=\left\{\tau(X, \Delta) \mid(X, \Delta) \in \mathcal{D}_{n}(\epsilon, \delta)\right\} .
$$

TheOrem 2.13 ([DiC17, Corollary 3.6]). Fix a positive integer $n$ and three positive real numbers $\epsilon, \delta$ and $\eta$. Then the set $\mathcal{T}_{n}(\epsilon, \delta) \cap[\eta, 1]$ is a finite set.

Applying this theorem in our situation, we obtain Corollary 2.15. To state this, we first need to introduce the notation $\mathcal{P}_{n}$.

Definition 2.14. Fix a positive integer $n$. We define $\mathcal{P}_{n}$ to be the set of pairs $(Y, L)$ such that

(i) $Y$ is a normal projective variety of dimension $n$,

(ii) $L$ is a base-point-free big Cartier divisor.

Corollary 2.15. Fix a positive integer $n$ and a positive real number $\eta$. Then the set

$$
\left\{a(Y, L) \mid(Y, L) \in \mathcal{P}_{n}\right\} \cap[\eta, \infty)
$$

is a finite set.

Proof. We may assume $\eta \leqslant 1 / 4(n+1)$. First, we show that the set

$$
\left\{a(Y, L) \mid(Y, L) \in \mathcal{P}_{n}\right\} \cap\left[\eta, \frac{1}{2}\right]
$$

is a finite set. Take $(Y, L) \in \mathcal{P}_{n}$ and assume $a(Y, L) \in\left[\eta, \frac{1}{2}\right]$. Note that $a\left(Y, \frac{1}{2} L\right)=2 a(Y, L) \in$ $[2 \eta, 1]$. By taking a resolution, we may assume that $Y$ is smooth. In this case, $a\left(Y, \frac{1}{2} L\right)=$ $\tau\left(Y, \frac{1}{2} L\right)$. Replacing $L$ by a general element in $|L|$, we may assume that $L$ is irreducible and 


\section{D. HACON AND C. JiAng}

smooth. Moreover, $\left(Y, \frac{1}{2} L\right)$ is $\frac{1}{2}$-lc and $K_{Y}+\frac{1}{2} L$ is pseudo-effective, that is, $\left(Y, \frac{1}{2} L\right) \in \mathcal{D}_{n}\left(\frac{1}{2}, \frac{1}{2}\right)$. This implies that the set

$$
\left\{a\left(Y, \frac{1}{2} L\right) \mid(Y, L) \in \mathcal{P}_{n}\right\} \cap[2 \eta, 1]
$$

is finite by Theorem 2.13, and so is $\left\{a(Y, L) \mid(Y, L) \in \mathcal{P}_{n}\right\} \cap\left[\eta, \frac{1}{2}\right]$.

Then we show that the set

$$
\left\{a(Y, L) \mid(Y, L) \in \mathcal{P}_{n}\right\} \cap\left[\frac{1}{2}, \infty\right)
$$

is a finite set. Take $(Y, L) \in \mathcal{P}_{n}$ and assume $a(Y, L) \geqslant \frac{1}{2}$. By taking a resolution, we may assume that $Y$ is smooth. By [LTT14, Proposition 2.10], we have $a(Y, L) \leqslant n+1$. Now we consider $(Y, 2(n+1) L) \in \mathcal{P}_{n}$. Note that $a(Y, 2(n+1) L)=(1 / 2(n+1)) a(Y, L)$, hence $a(Y, 2(n+1) L) \in$ $\left[1 / 4(n+1), \frac{1}{2}\right]$. By the first step, $a(Y, 2(n+1) L)$ belongs to a finite set. Hence $a(Y, L)$ belongs to a finite set.

\section{Proof of Theorem 1.1}

We prove the following proposition suggested by Lehmann.

Proposition 3.1. Fix a positive real number $t$. Let $X$ be a smooth projective variety and $L$ a big and semiample $\mathbb{Q}$-divisor. Then there is a bounded family $\mathcal{U}$ of subvarieties of $X$ such that any subvariety $Y$ not contained in $\mathbf{B}_{+}(L)$, with $a(Y, L)>t$, is dominated by some members $Z$ of $\mathcal{U}$ such that $a(Z, L)=a(Y, L)$.

Proof. Note that for a subvariety $Y$ not contained in $\mathbf{B}_{+}(L)$, the restriction $\left.L\right|_{Y}$ is nef and big, and so $a(Y, L)$ is well defined. Therefore, we will only consider subvarieties not contained in $\mathbf{B}_{+}(L)$. Replacing $L$ by some multiple, we may assume that $L$ is a base-point-free Cartier divisor. We construct $\mathcal{U}$ inductively by increasing induction on the dimension of $Y$.

For a subvariety $Y$ with $a(Y, L)>t$ and $\operatorname{dim} Y=1$, it is easy to see that $Y$ is a rational curve with

$$
\operatorname{deg}_{Y}(L)=Y \cdot L=\frac{2}{a(Y, L)}<\frac{2}{t} .
$$

By Lemma 2.4, such $Y$ form a bounded family $\mathcal{U}_{1}$.

Suppose that we have constructed a bounded family $\mathcal{U}_{i}$ of subvarieties such that every subvariety $Y$ with $a(Y, L)>t$ and $\operatorname{dim} Y \leqslant i$ is dominated by some members $Z$ of $\mathcal{U}$ such that $a(Z, L)=a(Y, L)$. We construct $\mathcal{U}_{i+1}$ as follows. Suppose that $Y$ is an $(i+1)$-dimensional subvariety satisfying $a(Y, L)>t$. By taking a resolution, we may assume that $Y$ is smooth. Proposition 2.3 shows that either

(1) $Y$ is covered by proper subvarieties $Z$ with $a(Z, L)=a(Y, L)$ or

(2) $Y$ is birational to a $\mathbb{Q}$-factorial terminal Fano variety $Y^{\prime}$ of Picard number 1.

In case $(1)$, by induction, $Z$ is dominated by some members $Z^{\prime}$ of $\mathcal{U}_{i}$ such that $a\left(Z^{\prime}, L\right)=$ $a(Z, L)$, and so is $Y$.

In case (2), by taking a resolution, we may assume that $\phi: Y \rightarrow Y^{\prime}$ is a morphism. By the proof of [LTT14, Proposition 4.6], we have $K_{Y^{\prime}}+a(Y, L) \phi_{*}\left(\left.L\right|_{Y}\right) \equiv 0$.

We define constants $c_{0}<1$ and $w>2$ as follows: since $L$ is base-point free, we know that the set

$$
\{a(Z, L) \mid Z \text { is a subvariety of } X\} \cap(t, \infty]
$$




\section{ON FUJITA INVARIANTS}

is finite by Corollary 2.15. Hence, we may take a rational number $c_{0}<1$ such that the set

$$
\{a(Z, L) \mid Z \text { is a subvariety of } X\} \cap\left[c_{0} a\left(Z^{\prime}, L\right), a\left(Z^{\prime}, L\right)\right)
$$

is empty for any subvariety $Z^{\prime}$ with $a\left(Z^{\prime}, L\right)>t$. Take $w=1 /\left(1-c_{0}\right)$. We may assume $w>2$ by taking $c_{0}>\frac{1}{2}$ in the definition.

$$
\begin{aligned}
& \text { If }\left(-K_{Y^{\prime}}\right)^{i+1} \leqslant(w(i+1))^{i+1} \text {, then } \\
& \qquad\left(\left.L\right|_{Y}\right)^{i+1} \leqslant\left(\phi^{*} \phi_{*}\left(\left.L\right|_{Y}\right)\right)^{i+1}=\left(\phi_{*}\left(\left.L\right|_{Y}\right)\right)^{i+1} \leqslant \frac{(w(i+1))^{i+1}}{a(Y, L)^{i+1}}<\frac{(w(i+1))^{i+1}}{a(X, L)^{i+1}},
\end{aligned}
$$

where the first inequality holds because by the negativity lemma, $\phi^{*} \phi_{*}\left(\left.L\right|_{Y}\right)-\left.L\right|_{Y}=E \geqslant 0$ and hence

$$
\left(\left.L\right|_{Y}\right)^{i+1-j}\left(\phi^{*} \phi_{*}\left(\left.L\right|_{Y}\right)\right)^{j}=\left(\left.L\right|_{Y}\right)^{i-j}\left(\phi^{*} \phi_{*}\left(\left.L\right|_{Y}\right)-E\right)\left(\phi^{*} \phi_{*}\left(\left.L\right|_{Y}\right)\right)^{j} \leqslant\left(\left.L\right|_{Y}\right)^{i-j}\left(\phi^{*} \phi_{*}\left(\left.L\right|_{Y}\right)\right)^{j+1}
$$

for $j=0,1, \ldots, i$, and where we have use the fact that $\phi_{*}\left(\left.L\right|_{Y}\right)$ is nef since $\rho\left(Y^{\prime}\right)=1$. By Lemma 2.4, such $Y$ form a bounded family $\mathcal{U}_{i+1}^{\prime}$.

Now, we assume $\left(-K_{Y^{\prime}}\right)^{i+1}>(w(i+1))^{i+1}$. By Proposition 2.8, for a general point $p \in Y^{\prime}$, there exists an effective $\mathbb{Q}$-divisor $\Delta_{p}^{\prime} \sim_{\mathbb{Q}}-\frac{1}{w} K_{Y^{\prime}}$ such that $V_{p}^{\prime} \subset \operatorname{Nklt}\left(Y^{\prime}, \Delta_{p}^{\prime}\right)$ is the minimal exceptional non-klt center containing $p$. Note that by Lemma 2.9 and the inequality $w>2$, we have $\operatorname{dim} V_{p}^{\prime}>0$. Let $\nu: \tilde{V}_{p}^{\nu} \rightarrow V_{p}^{\prime}$ be the normalization. For any $\mathbb{Q}$-Cartier divisor $G$ on $V_{p}^{\prime}$, we write $\left.G\right|_{\tilde{V}_{p}^{\nu}}=\nu^{*} G$. By Theorem 2.6, there is an effective $\mathbb{Q}$-divisor $\Delta_{\tilde{V}_{p}^{\nu}}$ such that

$$
\left.\left(K_{Y^{\prime}}+\Delta_{p}^{\prime}\right)\right|_{\tilde{V}_{p}^{\nu}} \sim_{\mathbb{Q}} K_{\tilde{V}_{p}^{\nu}}+\Delta_{\tilde{V}_{p}^{\nu}} .
$$

Note that since $K_{Y^{\prime}}+a(Y, L) \phi_{*} L \equiv 0$, we have

$$
K_{\tilde{V}_{p}^{\nu}}+\Delta_{\tilde{V}_{p}^{\nu}}+\left.\left(1-\frac{1}{w}\right) a(Y, L) \phi_{*} L\right|_{\tilde{V}_{p}^{\nu}} \sim_{\mathbb{Q}} 0
$$

Let $V_{p}$ be the strict transform of $V_{p}^{\prime}$ on $Y$. Let $\tilde{V}_{p}$ be a common resolution of $\tilde{V}_{p}^{\nu}$ and $V_{p}$ with morphisms $f: \tilde{V}_{p} \rightarrow V_{p}$ and $g: \tilde{V}_{p} \rightarrow \tilde{V}_{p}^{\nu}$. Then

$$
\begin{aligned}
K_{\tilde{V}_{p}} & +\left(1-\frac{1}{w}\right) a(Y, L) f^{*}\left(\left.L\right|_{V_{p}}\right) \\
& =g^{*}\left(K_{\tilde{V}_{p}^{\nu}}+\Delta_{\tilde{V}_{p}^{\nu}}+\left.\left(1-\frac{1}{w}\right) a(Y, L) \phi_{*} L\right|_{\tilde{V}_{p}^{\nu}}\right)-g_{*}^{-1} \Delta_{\tilde{V}_{p}^{\nu}}+E \\
& \sim_{\mathbb{Q}}-g_{*}^{-1} \Delta_{\tilde{V}_{p}^{\nu}}+E,
\end{aligned}
$$

where $E$ is a $g$-exceptional $\mathbb{Q}$-divisor. Note that the $\mathbb{Q}$-divisor $-g_{*}^{-1} \Delta_{\tilde{V}_{p}^{\nu}}+E$ is not big. Hence $K_{\tilde{V}_{p}}+(1-(1 / w)) a(Y, L) f^{*}\left(\left.L\right|_{V_{p}}\right)$ is not big and therefore

$$
a\left(V_{p}, L\right) \geqslant\left(1-\frac{1}{w}\right) a(Y, L)=c_{0} a(Y, L) .
$$

By the definition of $c_{0}$, this implies that $a\left(V_{p}, L\right) \geqslant a(Y, L)$. Since $p$ is a general point, $Y$ is dominated by such $V_{p}$. By induction, $V_{p}$ is dominated by some members $Z$ of $\mathcal{U}_{i}$ such that $a(Z, L)=a\left(V_{p}, L\right) \geqslant a(Y, L)$. Hence $Y$ is also dominated by some members $Z$ of $\mathcal{U}_{i}$ such that $a(Z, L) \geqslant a(Y, L)$. By Proposition 2.1 , by taking general members, $Y$ is dominated by some members $Z$ of $\mathcal{U}_{i}$ such that $a(Z, L)=a(Y, L)$.

Hence we may take $\mathcal{U}_{i+1}=\mathcal{U}_{i} \cup \mathcal{U}_{i+1}^{\prime}$, and the proof is completed. 


\section{D. HACON AND C. JiAng}

Proof of Theorem 1.1. Take $t=a(X, L)$ in Proposition 3.1. There is a bounded family $\mathcal{U}$ of subvarieties of $X$ such that any subvariety $Y$ not contained in $\mathbf{B}_{+}(L)$ with $a(Y, L)>a(X, L)$ is dominated by some members $Z$ of $\mathcal{U}$ such that $a(Z, L)=a(Y, L)>a(X, L)$. By Theorem 2.2, there exists a proper closed subset $W \subset X$ such that any member $Z$ of the family $\mathcal{U}$ satisfying $a(Z, L)>a(X, L)$ is contained in $W$. Hence, any subvariety $Y$ with $a(Y, L)>a(X, L)$ is contained in $W$.

\section{ACKNOWLEDGEMENTS}

The essential ideas in this note originated during a visit of the second author to the University of Utah in February 2015. The second author is grateful for the hospitality. The authors would like to thank Brian Lehmann for useful comments.

\section{REFERENCES}

Amb98 F. Ambro, The locus of log canonical singularities, 1998, arXiv:math.AG/9806067.

Bir16 C. Birkar, Anti-pluricanonical systems on Fano varieties, 2016, arXiv:1603.05765.

DiC17 G. Di Cerbo, On Fujita's spectrum conjecture, Adv. Math. 311 (2017), 238-248; https://doi. org/10.1016/j.aim.2017.02.018.

HTT15 B. Hassett, S. Tanimoto and Yu. Tschinkel, Balanced line bundles and equivariant compactifications of homogeneous spaces, Int. Math. Res. Not. 2015 (2015), no. 15, 6375-6410; https: //doi.org/10.1093/imrn/rnu129.

Jia13 X. Jiang, On the pluricanonical maps of varieties of intermediate Kodaira dimension, Math. Ann. 356 (2013), no. 3, 979-1004; https://doi.org/10.1007/s00208-012-0869-y.

Kol92 J. Kollár, Adjunction and discrepancies, Flips and abundance for algebraic threefolds, A Summer Seminar on Algebraic Geometry (Salt Lake City, Utah, August 1991), Astérisque 211 (1992), 183-192.

Kol97 J. Kollár, Singularities of pairs, Algebraic Geometry (Santa Cruz, 1995), Proc. Sympos. Pure Math., vol. 62 (Amer. Math. Soc., Providence, RI, 1997), 221-287.

Lai16 C.-J. Lai, Bounding the volumes of singular Fano threefolds, Nagoya Math. J. 224 (2016), no. 1, 37-73; https://doi.org/10.1017/nmj .2016.21.

LTT14 B. Lehmann, S. Tanimoto and Yu. Tschinkel, Balanced line bundles on Fano varieties, J. reine angew. Math., published online on 20 January 2016, https://doi.org/10.1515/ crelle-2015-0084, to appear in print.

Sho93 V. V. Shokurov, 3-fold log flips, Russian Acad. Sci. Izv. Math. 40 (1993), no. 1, 95-202; https: //doi.org/10.1070/IM1993v040n01ABEH001862.

Sho94 , An addendum to the paper "3-fold log flips" [Russian Acad. Sci. Izv. Math. 40 (1993), no. 1, 95-202], Russian Acad. Sci. Izv. Math. 43 (1994), no. 3, 527-558; https://doi.org/10. 1070/IM1994v043n03ABEH001579.

Christopher D. Hacon hacon@math.utah.edu

Department of Mathematics, University of Utah, Salt Lake City, UT 84112, USA

Chen Jiang chen.jiang@ipmu.jp

Kavli IPMU (WPI), UTIAS, The University of Tokyo, Kashiwa, Chiba 277-8583, Japan 